\title{
Structure Functions of Bound Neutrons
}

\author{
Sebastian Kuhn \\ Old Dominion University, Norfolk, VA 23529
}

\begin{abstract}
We describe an experiment measuring electron scattering on a neutron bound in deuterium with coincident detection of a fast, backward-going spectator proton. Our data map out the relative importance of the pure PWIA spectator mechanism and final state interactions in various kinematic regions, and give a first glimpse of the modification of the structure function of a bound neutron as a function of its off-shell mass. We also discuss a new experimental program to study the structure of a free neutron by extending the same technique to much lower spectator momenta.
\end{abstract}

Keywords: deuterium, off-shell, neutron, structure functions

PACS: 24.85.+p, 25.30.-c, 21.45.+v

\section{INTRODUCTION}

The structure functions of the proton have been measured over a truly impressive kinematic range, with high precision. These data have given insight into the internal quark structure of the proton and into the properties of QCD. Similar data for the neutron are highly desirable, but are lacking due to the absence of a free neutron target (or beam) of sufficient density. Instead, data taken on the deuteron are used to infer information on the neutron structure; however, because of Fermi motion, binding effects [1], possible modifications of bound nucleons in the medium $[2,3]$ and non-nucleonic degrees of freedom in deuterium like 6-quark configurations [4, 5], this extraction is non-trivial and model-dependent, especially at high $x$ and in the resonance region.

One possible approach to this problem is a detailed study of the effect of binding on nucleon structure. If we can model the structure modifications of a bound neutron confidently, we can correct the deuteron data and extract neutron information. For comparison with various models of binding effects, it is useful to emphasize scattering off a nucleon which is far off its mass shell. This can be done in the case of deuterium by "tagging" the struck neutron with a fairly high-momentum backward-moving proton. While on average the nucleons in deuterium are only loosely bound, if we require a "spectator" momentum of at least $300 \mathrm{MeV} / \mathrm{c}$, we can emphasize the part of the deuteron wave function corresponding to short distances, where binding effects are largest. This is the goal of the "Deeps" experiment, which studied the reaction $\mathrm{D}\left(e, e^{\prime} p_{s}\right) \mathrm{X}$. The experiment and some of its results are discussed in the next section.

A more direct approach would use the same technique - spectator proton tagging to select neutrons which are only weakly bound, close to their mass shell, and little affected by final state interactions. The additional advantage of measuring the spectator proton kinematics is that one can correct for the Fermi motion of the struck neutron. This method, "Barely Off-shell NUcleon Scattering", is the goal of the "BONUS" program which will be discussed in the last section. 


\section{THE “DEEPS” EXPERIMENT}

The data for the Deeps experiment were collected in spring 2002 with a $5.7 \mathrm{GeV}$ electron beam $(6-9 \mathrm{nA}$ current) in experimental hall B of the Thomas Jefferson National Accelerator Facility (Jefferson Lab, formerly CEBAF). The scattered electrons and the backward-moving protons were detected with the CEBAF Large Acceptance Spectrometer (CLAS) [6]. Electrons were identified using Cherenkov counters and by matching their momentum (measured by three layers of drift chambers) to the energy deposited in the electromagnetic calorimeter. The large acceptance of CLAS allowed us to collect data simultaneously from the quasi-elastic to deep inelastic kinematics $(W<2.7 \mathrm{GeV})$ and over a range in momentum transfer of $1.2 \mathrm{GeV}^{2} \leq Q^{2} \leq 5.5 \mathrm{GeV}^{2}$. Coincident protons were detected at angles up to $140^{\circ}$ and with momenta from $0.3 \mathrm{GeV} / \mathrm{c}$ to $0.7 \mathrm{GeV} / \mathrm{c}$. The missing mass of the unobserved debris in $\mathrm{D}\left(e, e^{\prime} p_{s}\right) \mathrm{X}$ can be reconstructed from the electron and proton kinematics, without Fermi smearing or binding shifts. In the spectator picture, the usual Bjorken variable $x$ can also be corrected for the initial motion of the struck neutron.

Our main goal in this experiment was to test the spectator approximation in this reaction, where the backward-moving proton is an uninvolved spectator to the reaction that occurs on the off-shell neutron. We developed a simple PWIA model of the reaction in this framework, using the Paris wave function for the momentum distribution of the nucleons in the initial state. By correcting for acceptance, efficiency and backgrounds and by dividing out trivial kinematic factors, we can express our results as the product of the probability to find the proton with initial momentum $p_{s}, P\left(p_{s}\right)$, and a (possibly) modified structure function $F_{2 n}$ of the neutron, which depends on the relativistically rescaled variables $x$ or $W$ and $Q^{2}$, as well as (possibly) the off-shell mass of the struck neutron.

In the top half of Fig. 1, we show the product $P\left(p_{s}\right) \times F_{2 n}$ for a final state mass around $W=1.5 \mathrm{GeV}$ and $Q^{2} \approx 1.8 \mathrm{GeV}^{2}$, in two bins of $p_{s}$. While the agreement with our simple PWIA spectator picture is rather good at backward angles relative to the momentum transfer vector $\vec{q}\left(\cos \left(\theta_{p q}\right) \leq-0.3\right)$, the data are clearly rising above the model curve at angles around $90^{\circ}$, especially for higher proton momenta. This is in good agreement with models of final state interaction (FSI) effects based on the reinteraction of the debris from the struck neutron with the spectator proton [7]. The FSI effect is larger for large $W$, since one can expect more hadronic particles in the final state in this case. It is maximal for light cone fractions around $\alpha_{s} \approx 1$, which corresponds to angles $\theta_{p q}$ slightly forward of $90^{\circ}$. FSI are much reduced at very forward and very backward angles, as well as lower momenta. All of these general trends are confirmed by our data.

If we concentrate on the backward region $\cos \left(\theta_{p q}\right) \leq-0.3$, the spectator model works relatively well and we can extract the "off-shell" neutron structure function $F_{2 n}^{\text {eff }}$. Our results (lower part of Fig. 1) indicate that at the highest momenta (above $400 \mathrm{MeV} / \mathrm{c}$ ) there may be some depletion of $F_{2 n}^{\text {eff }}$ relative to the on-shell neutron structure function, especially at moderate to high $x$. However, a full calculation with proper inclusion of FSI effects is needed to quantify this statement. 

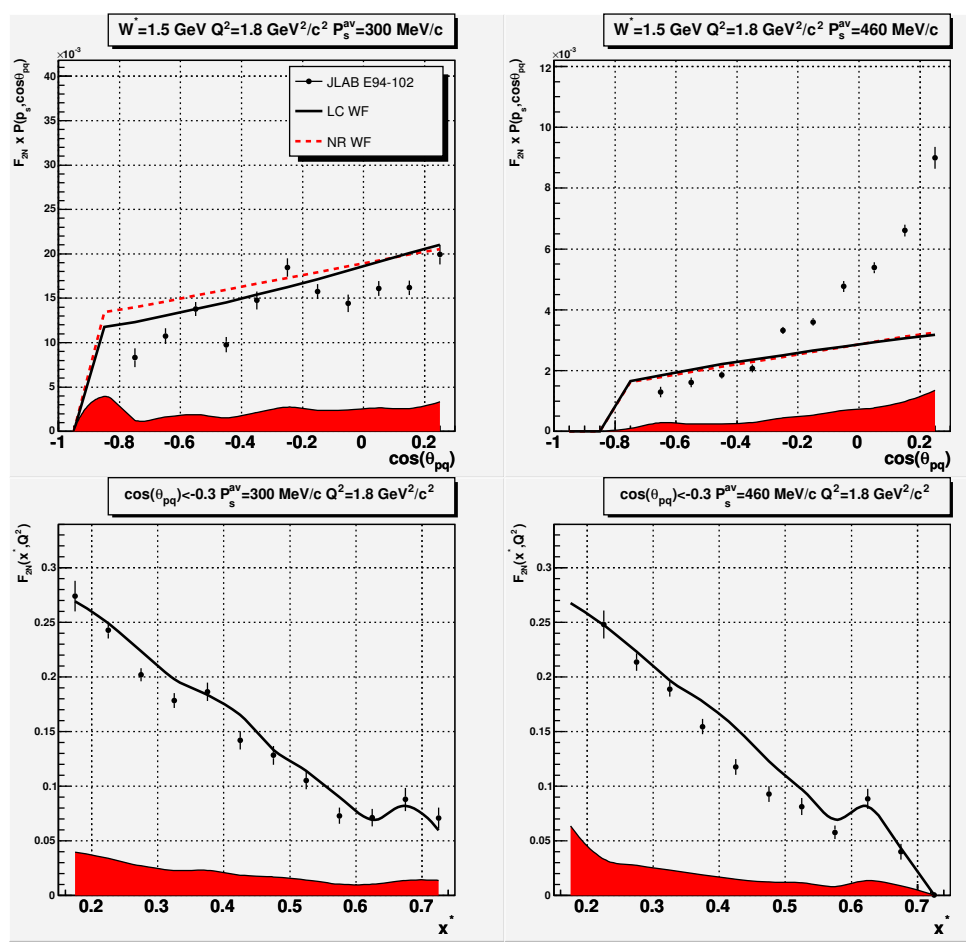

FIGURE 1. Angular distribution (reduced cross section vs. $\cos \left(\theta_{p q}\right)$ ) of "spectator" protons relative to the momentum transfer vector $\vec{q}$ (top two panels). The invariant mass of the unobserved final state of the struck neutron is in the region of the $S_{11}$ and $D_{13}$ resonances (around $1.5 \mathrm{GeV}$ ). The solid line is from a PWIA calculation within a strict spectator model, using a light cone wave function for the deuteron. The dashed line corresponds to the same calculation with a non-relativistic deuteron wave function. Bottom two panels: The effective structure function $F_{2 n}^{\text {eff }}$ of the neutron extracted from our data for two different momentum bins and backward spectator proton kinematics. The solid line shows a simple parametrization of the free neutron structure function.

\section{THE “BONUS” EXPERIMENT}

According to nearly all theoretical models of the $\mathrm{D}\left(e, e^{\prime} p_{s}\right) \mathrm{X}$ reaction (and corroborated by our "Deeps" data), both FSI and binding effects should be minimal if one selects spectator protons going backwards at momenta below $0.1 \mathrm{GeV} / \mathrm{c}$. The "BONUS" collaboration has prepared an experiment (to run in Fall 2005) that will use this method to extract the structure of the (nearly) free neutron, both in the resonance region and in the DIS region. In particular, we will measure the DIS structure function of the neutron out to $x=0.6$ without the nuclear uncertainties that become sizable around that point.

The scattered electron will again be detected with CLAS. The main new ingredient of this experiment is a novel recoil detector in the form of a radial time projection chamber (RTPC) surrounding a gaseous deuteron target. This detector is shown in Fig. 2. After exiting from the thin (4-6 mm diameter) target tube (50 $\mu \mathrm{m}$ Kapton), recoil protons with momenta down to $70 \mathrm{MeV} / \mathrm{c}$ traverse a buffer volume filled with helium where Möller electrons are curled up by a 3 Tesla solenoid field (not shown). The protons then enter a drift volume which extends from a radius of $3 \mathrm{~cm}$ to $6 \mathrm{~cm}$, surrounding the beam line. The ionization electrons released in this volume are drifted 


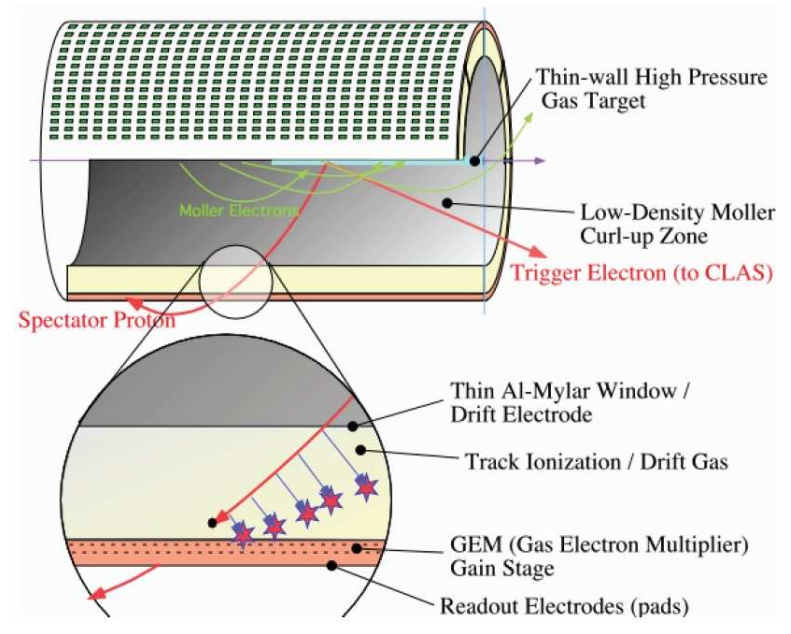

FIGURE 2. The general layout of the BoNuS RTPC. See text for explanation

to a series of three gaseous electron multiplier (GEM) foils that will amplify the signal more than 1000 fold. The signal is read out on a two-dimensional pattern of readout pads connected to continuously sampling ADCs (using the ALTRO chip developed at CERN). From the two spatial dimensions and the radial distance inferred from drift time, a three-dimensional track can be reconstructed. Proton momenta and identity can be reconstructed from the curvature of this track in the solenoid field and the energy deposited in the drift volume.

The detector system has been assembled and just passed a first engineering run with CLAS. The data to be collected in Fall will improve our knowledge of $F_{2 n}$, especially in the resonance region and at high $x$. At the same time, an extension of both experiments described here to $11 \mathrm{GeV}$ (after the anticipated Jefferson Lab upgrade) will generate a wealth of truly fundamental data not attainable anywhere else.

\section{ACKNOWLEDGMENTS}

We express our thanks to the CLAS collaboration and the technical staff at Jefferson Lab for their help and support.

\section{REFERENCES}

1. W. Melnitchouk, M. Sargsian, and M. I. Strikman, Z. Phys., A359, 99-109 (1997), nucl-th/ 9609048.

2. L. L. Frankfurt, and M. I. Strikman, Phys. Rept., 76, 215-347 (1981).

3. F. E. Close, R. L. Jaffe, R. G. Roberts, and G. G. Ross, Phys. Rev., D31, 1004 (1985).

4. C. E. Carlson, and K. E. Lassila, Phys. Rev., C51, 364-370 (1995), hep-ph/ 9401307.

5. C. E. Carlson, J. Hanlon, and K. E. Lassila, Phys. Rev., D63, 117301 (2001), hep-ph/9902281.

6. B. A. Mecking, et al., Nucl. Instrum. Meth., A503, 513-553 (2003).

7. C. Ciofi degli Atti, L. P. Kaptari, and B. Z. Kopeliovich, Eur. Phys. J., A19, 145-151 (2004), nucl-th/0307052. 